\title{
One-Year Clinical Outcomes of Biodegradable Polymer-Coated Sirolimus-Eluting Coronary Stent System in Patients with Coronary Artery Disease
}

\author{
Sridhar Kasturi*, Shailender Singh, Vijay Kumar Shanivaram, Kondal Rao Gollamandala, \\ Ganesh Mathan, Shiva Kumar Bandimida, Manikandhar Pendyala, Chandrashekar Challa \\ Sunshine Heart Institute, Hyderabad, India \\ Email: ^sridharkasturi@yahoo.com,manikandhar@yahoo.com
}

How to cite this paper: Kasturi, S., Singh, S., Shanivaram, V.K., Gollamandala, K.R., Mathan, G., Bandimida, S.K., Pendyala, M. and Challa, C. (2017) One-Year Clinical Outcomes of Biodegradable Polymer-Coated Sirolimus-Eluting Coronary Stent System in Patients with Coronary Artery Disease. World Journal of Cardiovascular Diseases, 7, 233-242.

https://doi.org/10.4236/wjcd.2017.78022

Received: May 4, 2017

Accepted: August 5, 2017

Published: August 8, 2017

Copyright $\odot 2017$ by authors and Scientific Research Publishing Inc. This work is licensed under the Creative Commons Attribution International License (CC BY 4.0).

http://creativecommons.org/licenses/by/4.0/

\begin{abstract}
Background: The biodegradable polymer-coated sirolimus-eluting coronary stent (SES) system for the treatment of coronary artery disease (CAD) has been implanted in a real-world patient population. Aim: The present study was designed to evaluate the safety and performance of Metafor ${ }^{\mathrm{rm}}$ sirolimuseluting coronary stent (SES) system in consecutive patients with CAD in a real-world patient population. Methods: All the consecutive 251 patients who underwent percutaneous coronary interventions with the use of Metafor sirolimus-eluting stents were included in this study. Patients were clinically/telephonically followed-up at one-year from August 2015 to August 2016. We observed and analyzed major adverse cardiac event (MACE) defined as the aggregate of cardiac death, myocardial infarction (MI), and any target lesion revascularization (TLR) at one-year. Also, stent thrombosis (ST) was observed at one-year patients follow-up. Results: We collected and analyzed the data for 251 patients. Out of 251 participants, 191 (76.1\%) were males, 95 (37.8\%) patients had diabetes, 117 (46.6\%) had ST-segment-elevation myocardial infarction (STEMI), and 134 (53.4\%) had hypertension. Mean patient age was $56.35 \pm 11.23$ years. A total of 295 lesions were treated, out of which $13.6 \%$ of the lesions were the long lesion of $\geq 40 \mathrm{~mm}$. At one-year, MACE occurred in 4 (1.6\%) of 251 patients, consisting of $2(0.8 \%)$ cardiac deaths, $2(0.8 \%) \mathrm{MI}$, and $0(0 \%)$ TLR. Stent thrombosis was reported in one patient $(0.4 \%)$. Conclusions: This retrospective data demonstrated excellent safety and performance of Metafor SES in a "real-world" consecutive CAD patient, indicating low rates of MACE and ST at one-year follow-up.
\end{abstract}




\section{Keywords}

Drug-Eluting Stents, Cobalt-Chromium, Hybrid Cell Design, Percutaneous Coronary Intervention

\section{Introduction}

Drug-eluting stents (DES) are broadly established for treatment of coronary artery disease $(\mathrm{CAD})$ patients undergoing percutaneous coronary intervention (PCI) [1] [2] [3]. The DES has been used in diverse real-life clinical practice due to their ability to reduce restenosis rates and the need for repeat revascularization [4] [5]. However, durable polymers in first-generation DES were linked with inflammation, vascular hypersensitivity reaction, late and very late stent thrombosis (ST) [5]. Therefore, efforts to prevent these risks have promoted the clinicians and stent industry to develop DES with biodegradable polymers with improved stent platforms, effective anti-restenosis drug, and polymer carriers [4]. Additionally, biodegradable polymers provide controlled release of antirestenotic agents and slow degradation of the coating, which would have enhanced the safety and performance of DES [2] [6]. Thus, DES such as Metafor (CE approved) Sirolimus-eluting Coronary Stent (SES) (Meril Life Sciences Pvt. Ltd., Vapi, India) has been developed, which would reduce the risk of target lesion revascularisation (TLR) and stent thrombosis (ST).

Metafor SES uses a formulation of low dose sirolimus $\left(1.25 \mu \mathrm{g} / \mathrm{mm}^{2}\right)$ timed to elute in approximately 30 - 40 days from a biodegradable polymer base which degrades simultaneously. Several studies of "limus family" have demonstrated excellent safety and performance in CAD patients in a real-world setting [7] [8] [9] [10].

In this retrospective study, we analyzed the 12 months results for 251 patients who participated in the study and were treated with Metafor. The primary endpoint was a major adverse cardiac event (MACE) at one-year defined as the aggregate of cardiac death, myocardial infarction (MI), and target lesion revascularisation (TLR). The ST was also analyzed in this study. The present "Real-Life" study was undertaken to evaluate safety and performance of using Metafor ${ }^{\mathrm{rm}}$ stents in PCIs for the treatment of coronary artery disease (CAD) across a wide range of patients treated in routine clinical practice.

\subsection{Study Design and Patient Population}

This was a retrospective, single-center, observational, postmarketing study. We retrospectively collected and analyzed the data for 251 patients at the Sunshine Heart Institute, Secunderabad, Hyderabad, Telangana, India. The study population included 251 patients, with single or multiple restenotic lesion(s) treated with the Metafor SES. This was a single-center study of consecutive patients who underwent stenting for the management of CAD using Metafor SES. The study 
procedure, data collection, and analysis were performed for all study participants. The study was conducted in accordance with the Helsinki declaration and informed consent was obtained from all patients. This study was approved by Independent Ethics Committee (IEC). The clinical/telephonic follow-up was performed at the following time points: 6 months and 12 months after discharge.

\subsection{Device Description and Interventional Procedure}

The Metafor SES (Meril Life Sciences Pvt. Ltd., Vapi, India) is built on NexGen ${ }^{\text {Tx }}$, an ultrathin $(65 \mu \mathrm{m})$ cobalt-chromium platform with a unique cell design comprising of an intelligent mix of open and close cells. The SES is coated with 1.25 $\mu \mathrm{g}$ sirolimus $/ \mathrm{mm}^{2}$ of stent surface area. The biodegradable polymer, which acts as a drug carrier, is a thin copolymer formulation combining two biodegradable components namely poly-L-lactic acid (PLLA) and poly(lactic-co-glycolic acid) (PLGA). The Metafor SES is available in sizes of 8, 13, 16, 19, 24, 29, 32, 37, 40, 44 , and $48 \mathrm{~mm}$ lengths and diameters of 2.00, 2.25, 2.50, 2.75, 3.00, 3.50, 4.00, and $4.50 \mathrm{~mm}$.

Coronary interventional procedures were carried out as per the cardiologist's discretion. Every part of the postoperative medical care, together with dual antiplatelet therapy and statins, were analyzed as per the cardiologist's discretion. Dual antiplatelet therapy (DAPT) including a loading dose of aspirin as per investigator's discretion was received by all patients. Aspirin was given along with clopidogrel bisulfate. All patients were maintained on a daily therapeutic dose of $75 \mathrm{mg}$ of clopidogrel bisulfate for a minimum of 1-year. Aspirin $\geq 75 \mathrm{mg}$ was continued throughout the length of the clinical investigation following index procedure, followed by lifelong aspirin treatment.

The following data were collected in this study: 1) Patient demographic details; 2) Severity of disease; 3) Medical history; 4) Lesion and procedure characteristics; 5) MACE. Patients were followed clinically/telephonically at 6 and 12 months.

\subsection{Definitions and Endpoints}

The primary endpoint of this study was to determine the rate of MACE. Major adverse cardiac event was defined as the aggregate of cardiac death, MI, and any TLR during the 12 months follow-up period after the index procedure. The ST was also evaluated in this study. The ST was classified according to the definitions of the Academic Research Consortium [11]. Procedural success was defined as successful stent placement at the desired position with $<30 \%$ residual stenosis by visual estimation, post-procedural TIMI 3 flow and no in-hospital target vessel failure (TVF). At follow-up, data were collected relating to current clinical status, any hospitalization after PCI and occurrence of any MACE and ST.

\subsection{Statistical Analysis}

Categorical data were presented as counts and percentages. All clinical and con- 
tinuous angiographic variables were presented as a mean \pm standard deviation. The time-to-event curve was presented as per the Kaplan-Meier method. All data were processed using the Statistical Package for Social Sciences, version 15 (SPSS, Chicago, IL, USA).

\section{Results}

\subsection{Baseline, Lesion and Procedural Characteristics}

The analysis consists of baseline clinical data and follow-up data collected at 6 months and 12 months. Mean patients age was $56.35 \pm 11.23$ years. A total of 251 patients data were collected and analyzed in this study. Out of 251 patients, $191(76.1 \%)$ were males, 95 (37.8\%) of patients had diabetes, and 134 (53.4\%) of patients were hypertensive. Among 251 patients, 117 (46.6\%) and 89 (35.4\%) patients were treated with a diagnosis of ST-segment-elevation myocardial infarction (STEMI) and unstable angina. A total of 17 patients underwent primary PCI. The baseline demographics of the patients are outlined in Table 1. Details of the lesion and procedural characteristics are outlined in Table 2. Also, 144 (48.8\%) lesions were located in the left anterior descending artery and $5(2.0 \%)$ were in bifurcations. A total of 295 lesions were treated with the Metafor SES (1.18 stents per lesion), and $13.6 \%$ of the lesions were treated with the Metafor

Table 1. Baseline characteristics of the study population.

\begin{tabular}{cc}
\hline Variables & Patients ( $\mathbf{n} \mathbf{2 5 1})$ \\
\hline Age (Years) & $56.35 \pm 11.23$ \\
Male & $191(76.1 \%)$ \\
Diabetes Mellitus & $95(37.8 \%)$ \\
Hypertension & $134(53.4 \%)$ \\
Chronic smoker & $19(7.6 \%)$ \\
Chronic alcohol use & $16(6.4 \%)$ \\
Family history of CAD & $17(6.8 \%)$ \\
Previous MI & $7(2.8 \%)$ \\
Previous PCI & $15(6 \%)$ \\
Previous CABG & $5(2 \%)$ \\
History of angina & $10(4 \%)$ \\
STEMI, n (\%) & $117(46.6 \%)$ \\
NSTEMI, n (\%) & $34(13.5 \%)$ \\
Unstable angina, n (\%) & $89(35.4 \%)$ \\
Stable angina & $11(4.4 \%)$ \\
Thrombolysed & $43(17.1 \%)$ \\
Primary PTCA & $17(6.8 \%)$ \\
\hline
\end{tabular}

CABG-Coronary artery bypass grafting, CAD—coronary artery disease, MI-myocardial infarction, NSTEMI-non-ST-segment-elevation myocardial infarction, PCI-percutaneous coronary intervention, PTCA-percutaneous transluminal coronary angioplasty, STEMI-ST-segment-elevation myocardial infarction. Data are presented as mean $\pm \mathrm{SD}$ or as number and percentage. 
Table 2. Lesion and procedural characteristics.

\begin{tabular}{|c|c|}
\hline Variables & $\begin{array}{l}\text { Patients }(n=251) \\
\text { Lesions }(n=295)\end{array}$ \\
\hline \multicolumn{2}{|l|}{ Severity of disease, $\mathrm{n}(\%)$} \\
\hline Single vessel disease & $131(52.2 \%)$ \\
\hline Double vessel disease & $94(37.5 \%)$ \\
\hline Triple vessel disease & $26(10.4 \%)$ \\
\hline Total no. of lesions treated with a study stent & 295 \\
\hline \multicolumn{2}{|l|}{ Target vessel locations } \\
\hline RCA & $89(30.2 \%)$ \\
\hline LAD & $144(48.8 \%)$ \\
\hline $\mathrm{LCx}$ & $57(19.3 \%)$ \\
\hline LM & $1(0.3 \%)$ \\
\hline Ramus & $4(1.4 \%)$ \\
\hline Stent per lesion & 1.18 \\
\hline Bifurcation lesion & $5(2.0 \%)$ \\
\hline$\%$ of stenosis (mean $\pm \mathrm{SD})$ & $88.95 \pm 7.6$ \\
\hline Total occlusion ( $100 \%$ stenosis) & $44(17.53 \%)$ \\
\hline post procedure TIMI III flow & $295(100 \%)$ \\
\hline Procedural success & $295(100 \%)$ \\
\hline Pre Balloon dilation & $187(63.4 \%)$ \\
\hline Post Balloon dilation & $199(67.5 \%)$ \\
\hline \multicolumn{2}{|l|}{ Stent length (mm), n (\%) } \\
\hline $12-19$ & $138(46.8 \%)$ \\
\hline $24-38$ & $117(39.7)$ \\
\hline $40-48$ & $40(13.6 \%)$ \\
\hline \multicolumn{2}{|l|}{ Stent diameter $(\mathrm{mm}), \mathrm{n}(\%)$} \\
\hline $2.5-3$ & $236(80 \%)$ \\
\hline $3.5-4.5$ & $59(20 \%)$ \\
\hline
\end{tabular}

DVD—double vessel diseases, LAD—-left anterior descending coronary artery, LCx-left circumflex coronary artery, LM-left main, RCA—right coronary artery, SVD—single vessel diseases, TVD—-triple vessel disease, TIMI-thrombolysis in myocardial infarction.

SES with length $\geq 40 \mathrm{~mm}$. Baseline lesion and procedural characteristics are mentioned in Table 2. An analysis of baseline angiography revealed that $52.2 \%$ of patients had single-vessel diseases (SVD) and $88.95 \%$ of patients had stenosis that was treated with Metafor SES stent. The most prevalent target vessel was left anterior descending (LAD) artery (48.8\%).

\subsection{Clinical and Angiographic Outcomes}

During 6-month follow-up, the primary endpoint occurred in $3(0.8 \%)$ of 251 patients, consisting of $2(0.8 \%)$ cardiac deaths, $1(0.4 \%) \mathrm{MI}$, and $0(0.0 \%)$ TLR, while at one-year, the primary endpoint occurred in 4 (1.6\%), consisting of 2 
$(0.8 \%)$ cardiac deaths, $2(0.8 \%)$ MI, and $0(0.0 \%)$ TLR. Also, ST was reported in one patient $(0.4 \%)$ at 6 -month as well as one-year follow-up. The summary of MACE during the one-year study period is presented in Table 3. The time-toevent analysis performed by Kaplan-Meier method was found to be $98 \%$ (Figure 1).

Table 3. Major adverse cardiac events at 6 months and 12 months follow-up.

\begin{tabular}{ccc}
\hline Events & 6-month Follow-up (N = 251) & 1-year Follow-up (N = 251) \\
\hline All cause death & $2(0.8 \%)$ & $3(1.2 \%)$ \\
Cardiac death, n (\%) & $1(0.4 \%)$ & $2(0.8 \%)$ \\
Non-cardiac death, n (\%) & $1(0.4 \%)$ & $1(0.4 \%)$ \\
MI, n (\%) & $1(0.4 \%)$ & $2(0.8 \%)$ \\
TLR, n (\%) & $0(0.0 \%)$ & $0(0.0 \%)$ \\
TVR, n (\%) & $0(0.0 \%)$ & $0(0.0 \%)$ \\
Stent thrombosis, n (\%) & $1(0.4 \%)$ & $1(0.4 \%)$ \\
MACE, n (\%) & $2(0.8 \%)$ & $4(1.6 \%)$ \\
\hline
\end{tabular}

MACE-major adverse cardiac event, MI-myocardial infarction, TLR-target lesion revascularization, TVR—-target vessel revascularization, ST—-stent thrombosis.

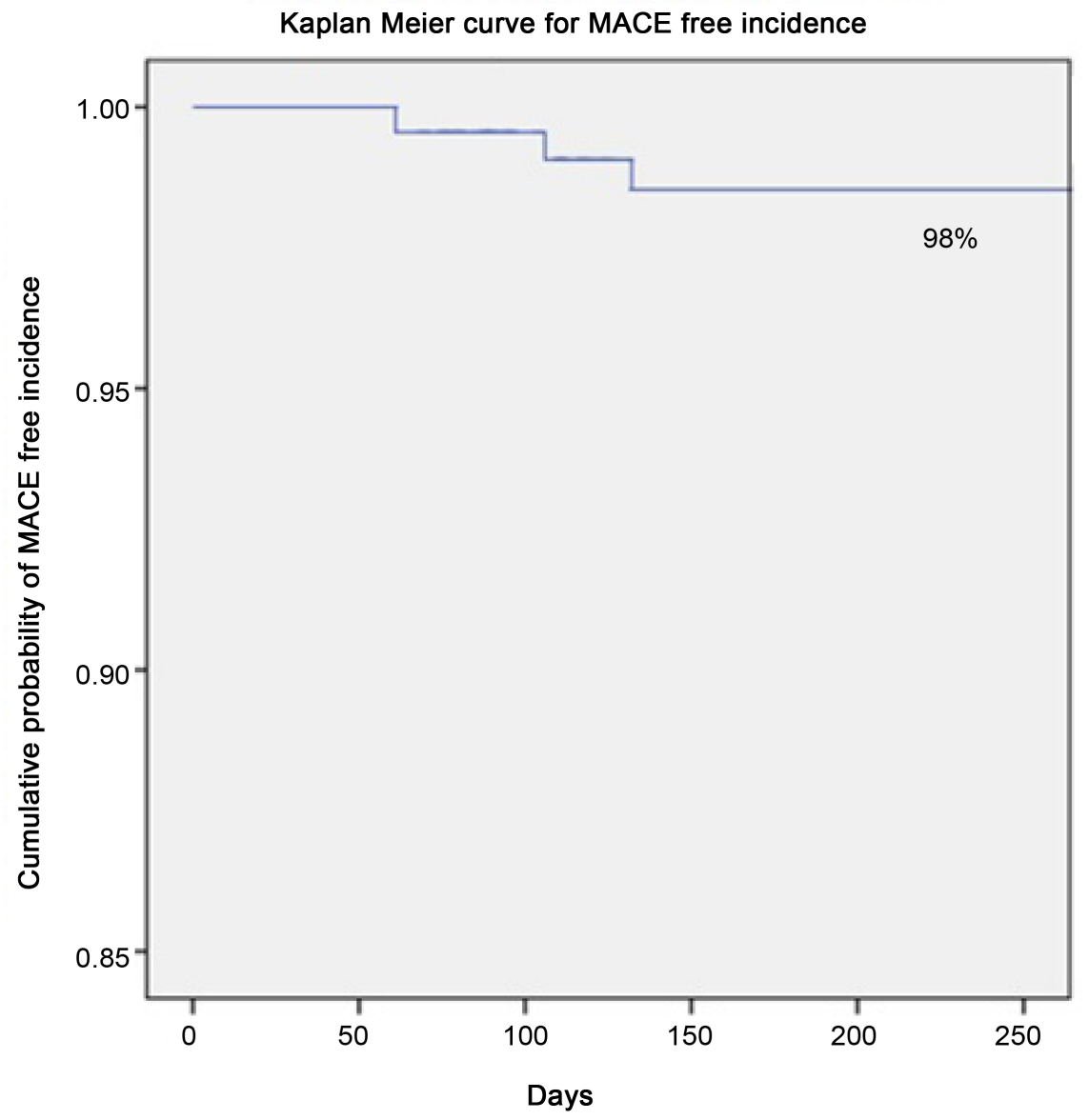

Figure 1. The time-to-event curve at one-year follow-up by Kaplan-Meier method. 


\section{Discussion}

In this retrospective study, the Metafor SES demonstrated excellent safety and performance in a "real-world", consecutive CAD patient at one-year follow-up. The study included complex coronary artery disease characteristics including patients with diabetes (37.8\%), hypertension (53.4\%), and unstable angina (35.4\%), which represents real clinical practice and allowing us to evaluate the Metafor SES in a "real-world" setting. This study included 251 consecutive patients requiring stenting for the management of CAD.

In this retrospective study, we collected and analyzed the data of Metafor SES with the aim to optimize the safety and performance of Metafor SES. The use of the biodegradable materials is considered to satisfy the concerns over late stent failure and minimize the risk of thrombus formation [2] [4].

The safety and performance of coronary stent platforms are varied on the following parameters: 1) The design of the stent; 2) The anti-proliferative drug; 3) Type of polymer. The safety and efficacy of "limus family" DES have been confirmed in several clinical studies [7] [8] [9] [10].

On another concern, strut thickness is a very significant parameter which causes local inflammation at the lesion site. Multiple studies have confirmed that the rate of restenosis was reduced according to strut thickness (strut thickness 50 $\mu \mathrm{m}$ vs $140 \mu \mathrm{m}$; a reduction in angiographic restenosis from $25.8 \%$ vs $15.0 \%$ ) [12] [13] [14]. The Metafor SES is based on an ultrathin cobalt-chromium platform; the thin struts enhance the device flexibility and improve device performance. The Metafor SES includes a hybrid cell design, the stent expands from middle to the edges and eliminates the classic dog-boning effect and thus provide excellent stent positioning.

Extensive research suggests that randomized controlled trials or first-in-man studies include a low-risk population and so outcomes of such trials cannot be assumed to "real-world" patients with greater risk coronary anatomy. A precise post-market surveillance study can provide important data of real-world patients treated in a routine clinical practice. Therefore, we analyzed this retrospective observational study to evaluate clinical outcomes of the Metafor SES in consecutive patients treated in a clinical setting.

The most important observations of this single-center, observational study is: 1) $100 \%$ procedural success rate; 2) A low rates of MACE (1.6\%) and ST (0.4\%) at one-year follow-up. At one-year, the primary endpoint (MACE) occurred in 4 (1.6\%) patients. This is significantly lower than the 5.0\% that was observed in the Nobori DES system $(\mathrm{p}=0.014)$ and the $4.5 \%$ that was noted in BioMatrix stent $(p=0.028)$ [15] [16]. Moreover, ST was reported in only one patient $(0.4 \%)$. This is comparable with other real-world study of Yukon Choice Flex stent which reported ST of $0.3 \%$ at one-year follow-up [4].

Also, at one-year follow-up period, the cardiac death in this retrospective study was $0.8 \%$. This is comparable with $0.4 \%$ patients in RESOLUTE Interna- 
tional trial [17].

Overall, these observations indicate the excellent performance of the Metaphor SES in a real-world setting in high-risk patients.

Moreover, the type of polymer (biodegradable vs durable polymer) and stent platform (cobalt-chromium vs platinum chromium) have resulted in significant improvement in clinical outcomes. In this retrospective study, the rates of MI and TLR were $0.8 \%$ and $0.0 \%$, respectively. These are lesser than $15.7 \%$ and $2.0 \%$ of MI and TLR that were observed in the Promus Element stent [18]. Therefore, the Metafor SES with thinner struts $(65 \mu \mathrm{m})$ has potential to improve the clinical results.

In present real-world population, lesser MACE rate (1.6\%) and ST incidence (0.4\%) at one-year follow-up demonstrated constructive clinical outcomes of the Metafor SES. However, longer follow-up of patients receiving the Metafor SES will be necessary to confirm long-term safety of this DES.

\section{Study Limitations}

Few limitations of this study: 1) Retrospective and observational design; 2) This was a non-randomised, single-arm study without comparison groups; 3) The absence of angiographic follow-up 4) Follow-up time of 12-month was relatively small. Therefore, long-term follow-up is essential to assess the factual event rates.

\section{Conclusion}

This retrospective data demonstrated excellent safety and performance of Metafor SES in a "real-world" consecutive CAD patient, indicating low rates of MACE and ST at one-year follow-up. Thus, Metafor SES can be implanted safely and routinely in PCI patients.

\section{Acknowledgements}

We thank all of the participating patients in this study.

\section{Disclosures}

The authors declare no conflict of interest.

\section{References}

[1] Simard, T., Hibbert, B., Chong, A.-Y., et al. (2012) Unprotected Left Main Coronary Artery Stenting with Zotarolimus (Endeavor) Drug-Eluting Stents. Catheterization and Cardiovascular Interventions, 80, E15-E22. https://doi.org/10.1002/ccd.23244

[2] Parsa, E., Saroukhani, S., Majlessi, F., et al. (2016) Biodegradable-Polymer Biolimus-Eluting Stents versus Durable-Polymer Everolimus-Eluting Stents at One-Year Follow-Up: A Registry-Based Cohort Study. Texas Heart Institute Journal, 43, 126 130. https://doi.org/10.14503/THIJ-14-4997

[3] Milewski, K., Gasior, P., Samborski, S., et al. (2016) Evaluation of Safety and Efficacy of NexGen-An Ultrathin Strut and Hybrid Cell Design Cobalt-Chromium Bare 
Metal Stent Implanted in a Real Life Patient Population-The Polish NexGen Registry. Advances in Interventional Cardiology, 12, 217-223. https://doi.org/10.5114/aic.2016.61642

[4] Hamon, M., Niculescu, R., Deleanu, D., et al. (2013) Clinical and Angiographic Experience with a Third-Generation Drug-Eluting Orsiro Stent in the Treatment of Single de Novo Coronary Artery Lesions (BIOFLOW-I): A Prospective, First-inMan Study. EuroIntervention, 8, 1006-1011. https://doi.org/10.4244/EIJV8I9A155

[5] Souza, C.F., El Mouallem, A.M., de Brito Junior, F.S., et al. (2013) Safety and Efficacy of Biolimus-Eluting Stent with Biodegradable Polymer: Insights from EINSTEIN (Evaluation of Next-Generation Drug-Eluting STEnt in Patients with Coronary Artery Disease) Registry. Einstein (Sao Paulo), 11, 350-356.

https://doi.org/10.1590/S1679-45082013000300015

[6] Goyal, B.K., Kalmath, B.C., Kawar, R., et al. (2013) Experience with BioMatrix BES and Other DES in All-Comers Setting: A Retrospective Overview. Indian Heart Journal, 65, 678-682. https://doi.org/10.1016/j.ihj.2013.10.014

[7] Natsuaki, M., Kozuma, K., Morimoto, T., et al. (2013) Biodegradable Polymer Biolimus-Eluting Stent versus Durable Polymer Everolimus-Eluting Stent: A Randomized, Controlled, Noninferiority Trial. Journal of the American College of Cardiology, 62, 181-190. https://doi.org/10.1016/j.jacc.2013.04.045

[8] Mehta, A.B., Chandra, P., Dalal, J., et al. (2013) One-Year Clinical Outcomes of BioMatrix $^{\mathrm{Tm}}$-Biolimus A9 ${ }^{\mathrm{Tm}}$ Eluting Stent: The E-BioMatrix Multicenter Post Marketing Surveillance Registry in India. Indian Heart Journal, 65, 593-599. https://doi.org/10.1016/j.ihj.2013.08.031

[9] Banker, D., Ahuja, A., Sanadhya, H., et al. (2016) Clinical Performance of Biodegradable Polymer-Coated Sirolimus-Eluting Stents in Unselected Real-World Population with Coronary Artery Disease: Results from the Multicenter CORE Registry. Minerva Cardioangiologica, 64, 9-14.

[10] Shetty, R., Vivek, G., Thakkar, A., et al. (2013) Experience with Biodegradable Polymer Coated Sirolimus-Eluting Coronary Stent System in "Real-Life" Percutaneous Coronary Intervention: 24-Month Data from the Manipal-s Registry. Journal of Clinical and Diagnostic Research, 7, 1959-1963.

[11] Cutlip, D.E., Windecker, S., Mehran, R., et al. (2007) Clinical end Points in Coronary Stent Trials a Case for Standardized Definitions. Circulation, 115, 2344-2351. https://doi.org/10.1161/CIRCULATIONAHA.106.685313

[12] Di Santo, P., Simard, T., Ramirez, F.D., et al. (2015) Does Stent Strut Design Impact Clinical Outcomes: Comparative Safety and Efficacy of Endeavor Resolute versus Resolute Integrity Zotarolimus-Eluting Stents. Clinical \& Investigative Medicine, 38, E296-E304. https://doi.org/10.25011/cim.v38i5.25686

[13] Pache, J., Kastrati, A., Mehilli, J., et al. (2003) Intracoronary Stenting and Angiographic Results: Strut Thickness Effect on Restenosis Outcome (ISAR-STEREO-2) Trial. Journal of the American College of Cardiology, 41, 1283-1288. https://doi.org/10.1016/S0735-1097(03)00119-0

[14] Kastrati, A., Mehilli, J., Dirschinger, J., et al. (2001) Intracoronary Stenting and Angiographic Results: Strut Thickness Effect on Restenosis Outcome (ISAR-STEREO) Trial. Circulation, 103, 2816-2821. https://doi.org/10.1161/01.CIR.103.23.2816

[15] Danzi, G.B., Chevalier, B., Urban, P., et al. (2012) Clinical Performance of a DrugEluting Stent with a Biodegradable Polymer in an Unselected Patient Population: The NOBORI 2 Study. EuroIntervention, 8, 109-116.

https://doi.org/10.4244/EIJV8I1A17 
[16] Urban, P., Valdés, M., Menown, I., et al. (2015) Outcomes Following Implantation of the Biolimus A9-Eluting Biomatrix Coronary Stent: Primary Analysis of the e-BioMatrix Registry. Catheterization and Cardiovascular Interventions, 86, 11511160. https://doi.org/10.1002/ccd.25892

[17] Neumann, F.-J., Widimsky, P., and Belardi, J.A. (2012) One-Year Outcomes of Patients with the Zotarolimus-Eluting Coronary Stent: RESOLUTE International Registry. EuroIntervention, 7, 1181-1188. https://doi.org/10.4244/EIJV7I10A189

[18] Lee, J.Y., Park, D.W., Kim, Y.H., et al. (2014) Comparison of Biolimus A9-Eluting (Nobori) and Everolimus-Eluting (Promus Element) Stents in Patients with De Novo Native Long Coronary Artery Lesions: A Randomized Long Drug-Eluting Stent V Trial. Circulation: Cardiovascular Interventions, 7, 322-329. https://doi.org/10.1161/CIRCINTERVENTIONS.113.000841

Submit or recommend next manuscript to SCIRP and we will provide best service for you:

Accepting pre-submission inquiries through Email, Facebook, LinkedIn, Twitter, etc. A wide selection of journals (inclusive of 9 subjects, more than 200 journals)

Providing 24-hour high-quality service

User-friendly online submission system

Fair and swift peer-review system

Efficient typesetting and proofreading procedure

Display of the result of downloads and visits, as well as the number of cited articles Maximum dissemination of your research work

Submit your manuscript at: http://papersubmission.scirp.org/

Or contactwjcd@scirp.org 